\title{
Ultra High Figure-of-Merit Mushroom Nanoantenna Array for Refractive Index Sensing
}

\author{
Nada Belal ${ }^{1}$, Mohamed Serry ${ }^{1}$, Gaurav Jayaswal ${ }^{2}$, Atif Shamim ${ }^{2}$, Ezzeldin A. Soliman ${ }^{1 *}$ \\ ${ }^{1}$ School of Sciences and Engineering, The American University in Cairo (AUC), New Cairo 11835, Egypt \\ ${ }^{2}$ Dept. of Electrical Engineering, King Abdullah University of Science and Technology (KAUST), Thuwal 23955-6900, KSA
}

(*) esoliman@aucegypt.edu

\begin{abstract}
In this paper, we introduce a developed version of mushroom nanoantenna sensor array suitable for refractive index sensing. The single element structure consists of goldsilica disks placed on top of a silica film backed by a gold layer. The whole structure is supported by silicon substrate, which is a passive substrate shielded from electromagnetic fields. The presence of the ground plan allows for isolating the proposed sensor from the medium underneath, which make it immune against parasitic variations in the supporting medium. The proposed developed mushroom sensor is fully optimized using a rigorous optimization algorithm. It shows a figure-of-merit (FoM) as high as 579, which is significantly better than other versions of mushroom sensors already presented in literature. The proposed device is fabricated using Electron Beam Lithography (EBL).
\end{abstract}

Index Terms - Nanaoantenna, refractive index sensing, plasmonics, localized surface plasmon resonance, mushroom sensor, EBL.

\section{INTRODUCTION}

Recently, the interaction between metals and optical waves has attracted much attention. When electromagnetic waves interact with the metallic surface of a nanostructure, the conduction electrons oscillate in two forms depending on the nanostructure geometry. The first form is Surface Plasmon Polaritons (SPP) in which the induced plasmons propagate along the metal/dielectric infinite interface. On the other hand, the induced plasmons oscillate locally to the finite surface of a nanoparticle, which is known as Localized Surface Plasmon Resonance (LSPR). As a result, the decay length of the electromagnetic field in SPP is larger than in LSPR. The shorter field decay length for LSPR leads to a higher sensitivity to refractive index changes on the surface. Consequently, LSPR based sensors are more suitable for bio-sensing applications $[1,2]$.

LSPR based sensors' performance is measured using three main quantities: Refractive Index Sensitivity (RIS), Quality Factor $(Q)$, and the Figure-of-Merit (FoM). RIS is the ratio between the resonance wavelength shift and the change in the surrounding refractive index, it has a unit of $\mathrm{nm} / \mathrm{RIU}$ (refractive index unit). $Q$ is a dimension less quantity defined as the ratio of the resonance wavelength to the Full Width at Half Maximum (FWHM). The third parameter is the best judge on the sensor performance as it is defined by the ratio of RIS to the FWHM. It has a unit of $\mathrm{RIU}^{-1}[3]$.

Most plasmonic works are performed using the noble metals especially silver and gold as their plasmon resonance is closer to the visible spectrum, which allows plasmon excitation by the standard optical sources. However, recent researches used poor metals, aluminum, copper and indium, and semiconductor materials for plasmonic sensing applications [4]. The sensing performance of indium nanodot placed on top of silica $\left(\mathrm{SiO}_{2}\right)$ substrate supported by silicon ( $\mathrm{Si}$ ) substrate is studied in [5]. It offers maximum RIS $696.3 \mathrm{~nm} / \mathrm{RIU}$ at around $550 \mathrm{~nm}$ resonance wavelength and maximum FoM of $27.8 \mathrm{RIU}^{-1}$ at $900 \mathrm{~nm}$.

As a silver based sensor, metal elliptical nanoring (MENR) arrays performance is investigated [6]. The MENR consists of silver nanorings arranged in rectangle lattice based on $\mathrm{SiO}_{2}$ substrate. The maximum RIS of 1200 $\mathrm{nm} / \mathrm{RIU}$ at $1670 \mathrm{~nm}$ resonance is for $30 \mathrm{~nm}$ ring width and $50 \mathrm{~nm}$ particle distance of the array. The maximum FoM achieved is $9.0 \mathrm{RIU}^{-1}$ for $140 \mathrm{~nm}$ ring width and particle distance of $910 \mathrm{~nm}$ under transverse polarization, i.e. incident light polarization is along the short axis of the elliptical nanoring.

Despite the fact that silver provides good sensing performance, gold is more chemically stable. Gold coated Silver NanoPrisms (GSNP) are studied at the visible spectrum. The prism edge length is about $30-40 \mathrm{~nm}$ and coated by 2-4 $\mathrm{nm}$ gold frame used to prevent truncation or rounding of SNP's sharp tips and edges. The prism height is $8-10 \mathrm{~nm}$. The reported bulk RIS is $425 \mathrm{~nm} / \mathrm{RIU}$ with FoM of $3 \mathrm{RIU}^{-1}$ [7]. Higher sensitivities are obtained in [8], the RIS of GSNP is theoretically enhanced to be $1133 \mathrm{~nm} / \mathrm{RIU}$ with FoM of $5.85 \mathrm{RIU}^{-1}$ for triangular gold nanoframe.

Bowtie and bowtie nanoring nantenna arrays' performance are studied in [9]. The sensor consists of gold bowtie with $200 \mathrm{~nm}$ side length, bowtie gap width of $40 \mathrm{~nm}$ and the hole size inside the bowtie varied from $20 \mathrm{~nm}$ to 50 $\mathrm{nm}$. The Au bowtie nanoring array with $40 \mathrm{~nm}$ thickness is placed on top of $\mathrm{SiO}_{2}$ substrate. The reported bulk RIS increases from $538 \mathrm{~nm} / \mathrm{RIU}$ to $881 \mathrm{~nm} / \mathrm{RIU}$ at $1400 \mathrm{~nm}$, which is about $63 \%$ enhancement compared to the solid bowtie. In [10], RIS, FoM of hollow gold and silica-gold nanoshells are studied. They are found to be $223.6 \mathrm{~nm} / \mathrm{RIU}$, 2.59 $\mathrm{RIU}^{-1}$ and $214.7 \mathrm{~nm} / \mathrm{RIU}, 2.5 \mathrm{RIU}^{-1}$, respectively.

Gold-silica-gold nanosandwich provide maximum RIS of $1550 \mathrm{~nm} / \mathrm{RIU}$ at $3492 \mathrm{~nm}$ resonance wavelength [11, 12]. But, it is clear that it has low FoM. Array of U-shaped gold nanostructures on an indium tin oxide (ITO) coated BK7 glass substrate is presented in [13]. The maximum RIS obtained is $170 \mathrm{~nm} / \mathrm{RIU}$, while the maximum FoM is 2.3 $\mathrm{RIU}^{-1}$. In [14], RIS of nanoholes made in silica supported 
gold films have been studied. It is reported that the higher RIS is $166 \mathrm{~nm} / \mathrm{RIU}$ for hole diameter to height aspect ratio of 150:30.

Mushroom gold-silica nanoantenna array proved to have good sensing performance. To the best of our knowledge the maximum FoM reported for such sensors is $108 \mathrm{RIU}^{-1}[15-$ 17]. In this paper, we present a developed mushroom nano antenna array sensor. Its structure dimensions are optimized using MATLAB to reach a maximum RIS of $1157 \mathrm{~nm} / \mathrm{RIU}$ at $1677 \mathrm{~nm}$ and maximum FoM $578.5 \mathrm{RIU}^{-1}$ and a $Q$ of 838.5, which are better than all other mushroom sensors reported in literature. The effect of varying each dimension on the reflectance spectrum is studied.

The paper is structured as follows. Section II presents our developed sensor. The modal field distribution, optimization process and the parametric study are presented in Section III, IV and V, respectively. A brief description of the fabrication process of the proposed device is outlined in Section VI. The paper is concluded in Section VII.

\section{DeVEloped Mushroom SENSOR}

Gold-silica mushroom is one of the simplest nanostructures used for refractive index sensing. By optimizing the dimensions of the mushroom sensor presented in [15] and with the insertion of a ground plane, an improved FoM is achieved. Fig. 1 shows the geometry of the proposed mushroom sensor, where the diameter of the silica and gold disks are donated by $D . H_{1}$ and $H_{2}$ are gold and silica disk height, respectively. $P$ is the array periodicity and $H_{3}$ is the silica film thickness. The optimum values of $\mathrm{D}, \mathrm{H}_{1}, \mathrm{H}_{2}, \mathrm{H}_{3}$ and $P$ are 220,100, 300, 300, and $1290 \mathrm{~nm}$, respectively. The sensor is supported by silicon, or any other material, substrate. A gold layer with a thickness of $100 \mathrm{~nm}$ is inserted in between the silica film and the silicon substrate.

Simulations are performed using the frequency-domain solver of CST Microwave Studio with periodic boundaries along the lateral directions. The air and silicon half-spaces from top and bottom sides, respectively, are assumed unbounded. One floquet mode is considered at both the upper and lower excitation ports. The dielectric function of gold is described according to Johnson and Christy from CST Microwave Studio material library. As for silica disk and film, their refractive index is set to 1.45 . The reflectance spectrum is calculated for two different values of surrounding medium refractive index, $n=1.33$ and $n=1.34$ (i.e. an index shift of 0.01). As shown in Fig. 2, the optimized sensor has resonance at $1677 \mathrm{~nm}$, with low FWHM of $2 \mathrm{~nm}$ and RIS of $1157 \mathrm{~nm} /$ RIU. As a result, it offers $Q$-factor of 838.5 and FoM as high as $578.5 \mathrm{~nm} / \mathrm{RIU}^{-1}$.

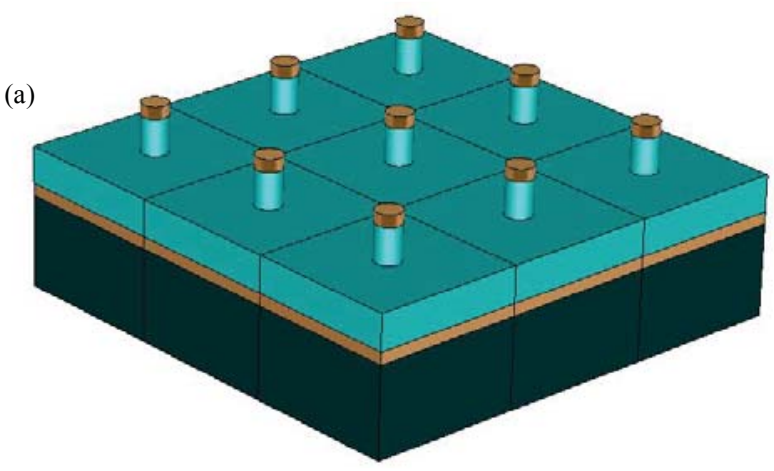

(b)

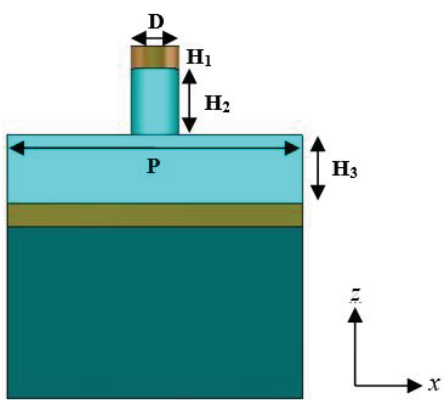

Fig. 1. Geometry of the proposed grounded mushroom sensor supported by silicon substrate: (a) perspective view of the array, and (b) cross-section view of the single element.

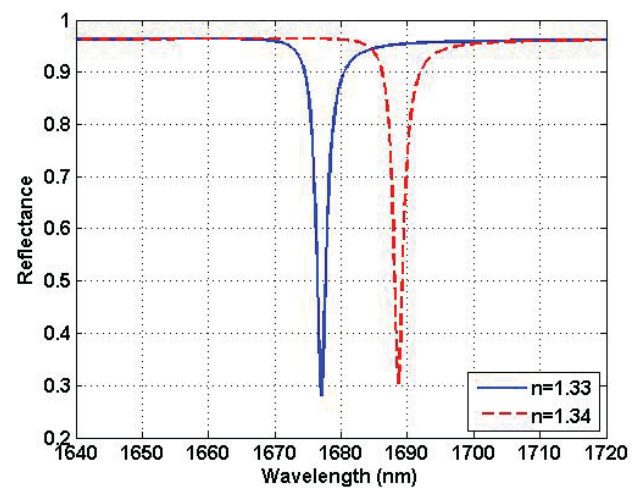

Fig. 2. Reflectance spectrum of the optimized developed mushroom nano antenna sensor array.

\section{DIMENSIONS OPTIMIZATION}

The proposed sensor is optimized by linking MATLAB with CST. The optimization algorithm adopted by "fmincon" function of MATLAB is proven to be more effective than genetic algorithm and "fminimax." However, its main weakness is that it uses single objective function. Since our problem is multi-objective, we use $\epsilon$-constraint method to scale the multi-objective problem into a single objective one [18]. Our main goal is to maximize FoM while RIS is kept higher than $1100 \mathrm{~nm} / \mathrm{RIU}$ and the reflectance level at resonance is kept less than 0.4 . Fig. 3 shows the enhancement of the FoM versus the iteration number of fmincon. 


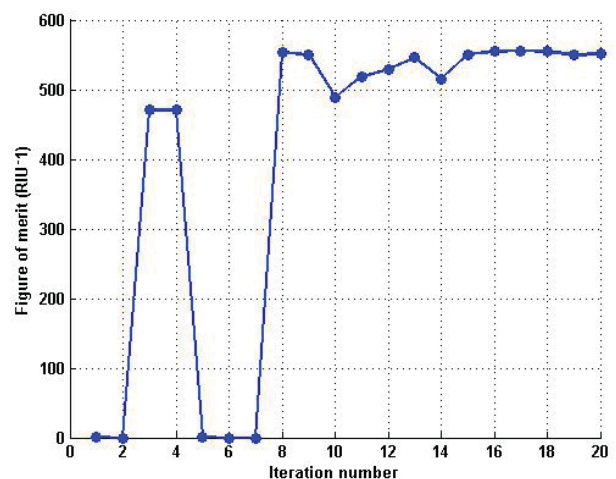

Fig. 3. Figure-of-Merit (FoM) versus fmincon iteration number.

It is clear that the FoM starts with a small value and gets improved as the iterations go on, which indicates the high efficiency of the selected optimization algorithm. It is worth mentioning that the FoM is very low at the dimensions of the lower and upper bounds given to the optimizer, which validates the selected optimization range. The optimizer converges to the optimum FoM value after the $20^{\text {th }}$ iteration, as shown in Fig. 3. The optimum set of dimensions is: $D=$ $220 \mathrm{~nm}, H_{1}=100 \mathrm{~nm}, H_{2}=300, H_{3}=300 \mathrm{~nm}$ and $P=1290$ nm.

The proposed developed mushroom sensor is compared with other mushroom sensors presented so far in literature. Table I list the different performance measures of the reported sensors together with the proposed one. It is clear that the current design is comparable with the previous ones from RIS point of view. However, it is way better than them from the points of view of both FWHM and FoM. Since the FoM can be considered as some sort of normalized sensitivity, it is more significant than the absolute sensitivity. Hence, the proposed design is fairly better than the previous ones.

TABLE I.

COMPARISON BETWEEN DIFFERENT MUSHROOM DESIGNS REPORTED IN LITERATURE TOGETHER WITH THE CURRENT DESIGN.

\begin{tabular}{cccccc} 
Design & $\begin{array}{c}\text { Resonance } \\
\text { wavelength } \\
\text { (nm) }\end{array}$ & $\begin{array}{c}\text { RIS } \\
(\mathbf{n m} / \mathbf{R I U})\end{array}$ & $\begin{array}{c}\text { FWHM } \\
(\mathbf{n m})\end{array}$ & $\boldsymbol{Q}$ & $\begin{array}{c}\text { FoM } \\
\left.\mathbf{R I U}^{-1}\right)\end{array}$ \\
\hline \hline$[\mathbf{1 5}]$ & 1552 & 948 & 140 & 11.1 & 6.8 \\
{$[\mathbf{1 6}]$} & 700 & 356 & - & - & - \\
{$[\mathbf{1 7}]$} & 1255 & 1015 & 9.5 & 135.6 & 108 \\
This & 1677 & 1157 & 2 & 838.5 & 578.5 \\
Work & & & & &
\end{tabular}

\section{MOdAl Field AT Resonance}

LSPR results in strong electric field confinement around the nanostructure. The regions where the near field is intensive are known as the "hot spots". Sensor sensitivity is enhanced when the contact volume between these hot spots and the analyte becomes bigger. Fig. 4 shows the modal

field distribution at the resonance wavelength of the proposed sensor. It is clear that the hot spots of the sensor are almost entirely within the surrounding analyte, which leads to high RIS as required. Only little portions from the hot spots lie within the supporting silica disk. Consequently, the etching in silica disk adds to the complexity of fabrication with no significant corresponding enhancement in the performance measures.

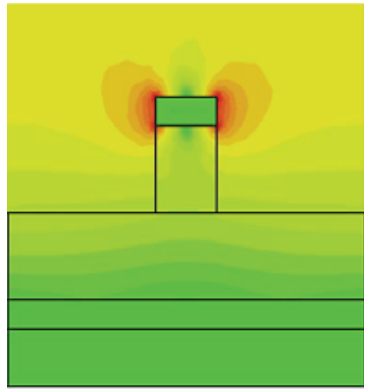

(a)

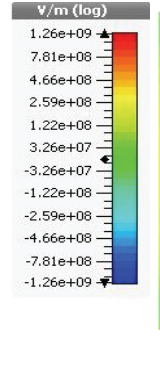

Fig. 4. Modal electric field magnitude distribution of the developed mushroom sensor at $1677 \mathrm{~nm}$ : (a) side view and (b) top view.

\section{PARAMETRIC STUDY}

The optimum dimensions are the central values around which a parametric study is performed. Sensing performance is studied for each parameter variation, while all other parameters are fixed.

\section{A. Gold disk diameter}

The resonance condition is that the length of the equivalent resonating dipole, $L_{\text {dip }}$ in Fig. 4(b), equals half of a guided wavelength, $\lambda_{g}$. This is because any increase or decrease in the length around this value results in a reduction in the electric field intensity within the hot-spots of the gold disk. The guided wavelength equals the freespace wavelength, $\lambda_{0}$, divided by the effective refractive index, $n_{\text {eff }}$, of the hybrid media forming the resonator. This can be written mathematically as follows:

$$
L_{\text {dip }}=\frac{\lambda g}{2}=\frac{\lambda_{0}}{2 n_{e f f}}
$$

As shown in Fig. 5 the resonance wavelength red shifts when the disk diameter increases. This can be explained as follows: the increase in $D$ means an increase in $L_{d i p}$, which results in an increase in $\lambda_{0}$ for the same $n_{\text {eff }}$ according to equation (1). As $D$ decreases, the reflectance level is up shifted to 0.4 which means that $60 \%$ of the incident power is not interacting with the analyte under test. The RIS has maximum value of $1157 \mathrm{~nm} / \mathrm{RIU}$ at the optimum point $D=$ $220 \mathrm{~nm}$.

\section{B. Gold disk height}

When the height of the gold disk increases the resonance wavelength slightly red shifts, and the interaction between 
the analyte and hot spots around the gold disk increases. Hence the RIS increases. However, the FWHM increases which leads to almost unchanged FoM for small variations in $H_{1}$. The red shift of the resonance wavelength due to the increase in $H_{1}$ is due to the corresponding increase in the effective refractive index of the hybrid medium forming the sensor. Consequently, the resonance wavelength has to increase, as shown in Fig. 6, to keep the same value for $L_{d i p}$ according to equation (1).

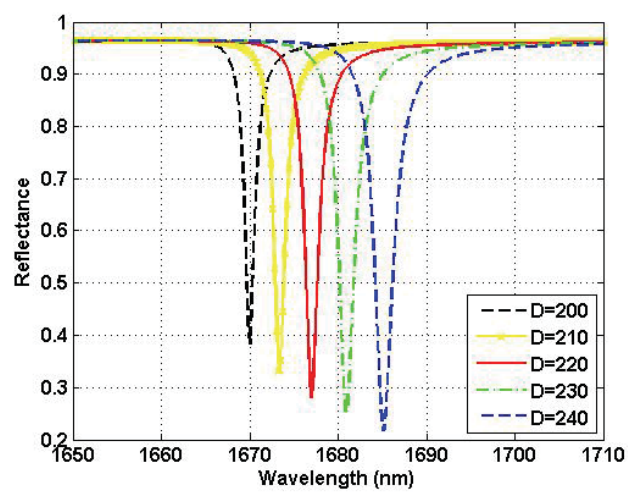

Fig. 5. Reflectance spectrum for different values of the gold disk diameter $D$.

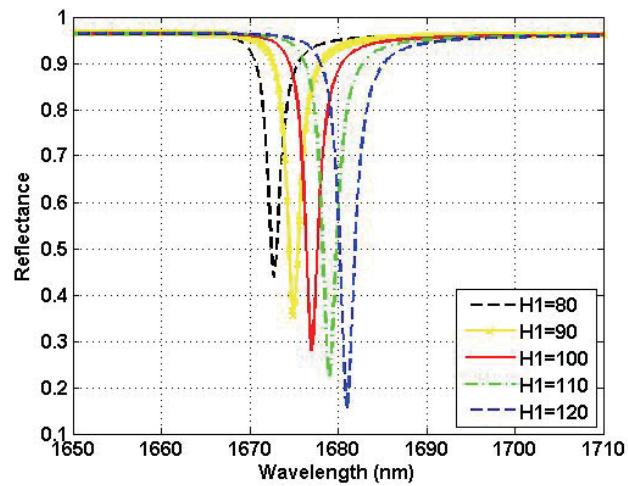

Fig. 6. Reflectance spectrum for different values of the gold disk thickness $H_{1}$.

\section{Silica disk height}

When silica disk height increases, the resonance dip down shifts leads to higher FWHM and lower FoM and $Q$. On the other hand, the RIS decreases as the disk height decreases and the reflectance level becomes 0.68 as shown in Fig. 7. Similar to $H_{1}$, the increase in $H_{2}$ results in an increase in $n_{\text {eff, }}$ which leads to an increase in $\lambda_{0}$ for the same $L_{\text {dip }}$.

\section{Silica film thickness}

As the thickness of the silica film increases, the resonance dip pulled down indicating more power interacting with the surrounding analyte, but the dip width (FWHM) increases which results in lower FoM and $Q$ values, i.e the sensing performance gets worse, as shown in
Fig. 8. Once more, the increase in $H_{3}$ gives rise to an increase in both $n_{\text {eff }}$ and $\lambda_{0}$, such that $L_{\text {dip }}$ remains the same.

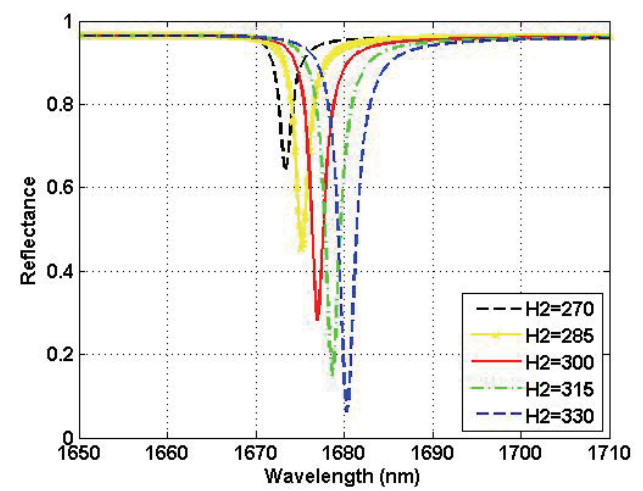

Fig. 7. Reflectance spectrum for different values of the silica disk height $\mathrm{H}_{2}$.

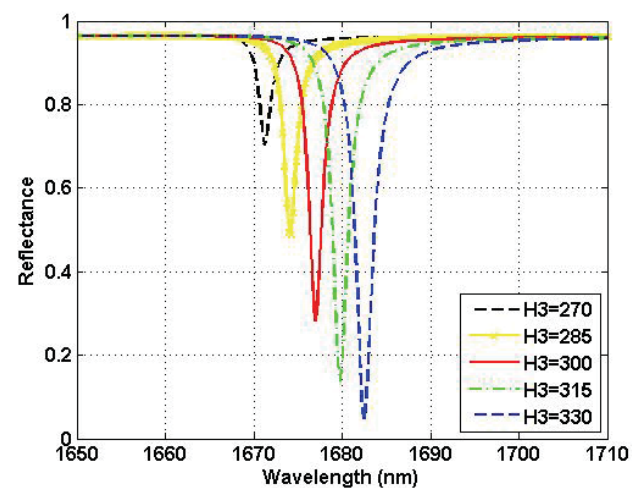

Fig. 8. Reflectance spectrum for different values of the silica film thickness $\mathrm{H}_{3}$.

\section{E. Array periodicity}

It is clearly observed that the reflectance spectrum is highly affected by the array periodicity. As $P$ increases the resonance wavelength increases, as shown in Fig. 9. Moreover, the reflectance level at resonance also shifts up as $P$ increases. The optimum value $P=1290 \mathrm{~nm}$ is selected to be a compromise between best sensor performance and accepted value for reflectance level.

\section{FABRICATION}

A $300 \times 300$ array of mushroom nantennas was fabricated starting from a Si substrate, and then building the device layers shown in Fig. 1(b), by: (1) e-beam evaporation of 100 $\mathrm{nm} \mathrm{Au}$ ground plan followed by (2) PECVD deposition of $600 \mathrm{~nm}$ silicon oxide layer, and then (3) e-beam evaporation of $100 \mathrm{~nm} \mathrm{Au}$ active layer. Then the top Au layer is patterned by EBL to form a pattern of 220 diameter disks with $1290 \mathrm{~nm}$ periodicity using PMMA A4 950 photoresist spinned at $2000 \mathrm{rpm}$ for 60 seconds to give an estimated thickness of 180-200 nm and EBL exposure dose of 1.2 $\mathrm{uC} / \mathrm{cm}^{2}$, as shown in Fig. 10. $100 \mathrm{~nm} \mathrm{Au}$ and $300 \mathrm{~nm}$ silicon 
oxide layers will be then dry etched in Ar-plasma using Oxford Instruments Plasmalab-100 and photoresist is stripped in $\mathrm{O}_{2}$ plasma to produce the final device structure shown in Fig. 10.

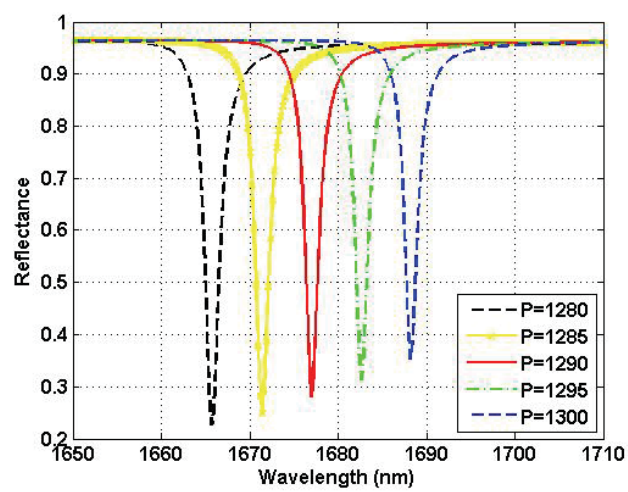

Fig. 9. Reflectance spectrum for different values of the array periodicity $P$.

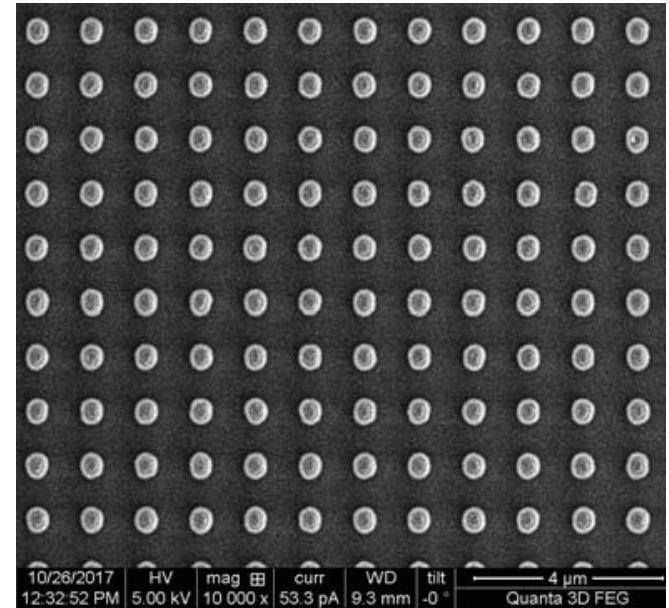

Fig. 10. SEM of the EBL patterned mushroom array.

\section{CONCLUSION}

A developed mushroom sensor is presented in this paper. The proposed sensor is rigorously optimized. The optimum design offers ultra-high FoM of 578.5 $\mathrm{RIU}^{-1}, Q$ of 838.5, and RIS of $1157 \mathrm{~nm} / \mathrm{RIU}$ at $1677 \mathrm{~nm}$. A parametric study showing the impact of varying the device's dimensions on its performance is presented. The proposed sensor is fabricated using electron beam lithography (EBL) technology. Preparation for measurements is currently going on.

\section{REFERENCES}

[1] S. Maier, Plasmonics. New York: Springer, 2007.

[2] J. Hammond, N. Bhalla, S. Rafiee, and P. Estrela, "Localized surface plasmon resonance as a biosensing platform for developing countries," Biosensors, vol. 4, no. 2, pp. 172-188, Jun. 2014.

[3] V. Chalishazar, "Nanostructured optical devices for biosensing applications," M.S. thesis, Dept. Elect. Eng., Oregon State University., Corvallis, Oregon, 2016
[4] Jianhua Zhou, Yangyang Wang, Li Zhang, Xuemeng Li, "Plasmonic biosensing based on non-noble-metal materials," Chinese Chemical Letters,2017 Available: http://dx.doi.org/10.1016/j.cclet.2017.09.003.

[5] X. Xu, X. Hu, X. Chen, Y. Kang, Z. Zhang, K. B. Parizi, and H.-S. P. Wong," Engineering a large scale indium nanodot array for refractive index sensing" ACS Applied Materials \& Interfaces, vol. 8, no.46, pp. 31871-31877, Nov.2016, DOI: 10.1021/acsami.6b11413.

[6] W. Lin, Q. Wang, Q. Li, A. Dong "The local surface plasmon resonance property and," refractive index sensitivity of metal elliptical nano-ring arrays", AIP Adv.,vol. 4, p. 117115, Nov. 2014.

[7] E. Martinsson, M.M. Shahjamali, K. Enander, F. Boey, C. Xue, D. Aili, B. Liedberg,"Local refractive index sensing based on edge goldcoated silver nanoprisms", J. Phys. Chem. C, vol. 117, pp. 2314823154, Oct. 2013.

[8] M. M. Shahjamali, M. Salvador, M. Bosman, D. S. Ginger, and C. Xue, " Edge-Gold-Coated Silver Nanoprisms: enhanced stability and applications in organic photovoltaics and chemical sensing", The Journal of Physical Chemistry $C$ vol. 118, no. 23,pp. 12459-12468, May 2014, DOI: 10.1021/jp501884.

[9] L.-W. Nien, B.-K. Chao, J.-H. Li, and C.-H. Hsueh, "Optimized sensitivity and electric field enhancement by controlling localized surface plasmon resonances for bowtie nanoring nanoantenna arrays," Plasmonics, vol. 10, no. 3, pp. 553-561, Nov. 2014.

[10] P. Tuersun, "Optimizing the figure of merit of gold nanoshell-based refractive index sensing," Optik - International Journal for Light and Electron Optics, vol. 127, no. 1, pp. 250-253, 2016.

[11] S. M. Kandil, I. S. E. Babli, and A. H. Badawi, "Study of the gap influence on highly sensitive plasmonic nanosandwich for refractive index sensing," 2016 10th European Conference on Antennas and Propagation (EuCAP), 2016.

[12] S. M. Kandil, I. S. El Babli and A. H. Badawi, "Tapered split ring nanosandwich for refractive index sensing," 2016 8th Cairo International Biomedical Engineering Conference (CIBEC), Cairo, 2016, pp. 56-59. DOI: 10.1109/CIBEC.2016.7836119

[13] N. Nehru, L. Yu, Y. Wei and J. T. Hastings, "Using U-shaped localized surface plasmon resonance sensors to compensate for nonspecific interactions," IEEE Transactions on Nanotechnology, vol. 13, no. 1, pp. 55-61, Jan. 2014. DOI: 10.1109/TNANO.2013.2287700

[14] F. Mazzotta, T. W. Johnson, A. B. Dahlin, J. Shaver, S.-H. Oh, and F. Höök, "Influence of the evanescent field decay length on the sensitivity of plasmonic nanodisks and nanoholes," ACS Photonics vol. 2 no. 2, pp. 256-262, 2015, DOI: 10.1021/ph500360d

[15] S. M. Kandil, T. A. Ali, S. Sedky, and E. A. Soliman, "Highly sensitive mushroom-shaped gold-silica nano antenna array for refractive index sensing," 2016 10th European Conference on Antennas and Propagation (EuCAP), 2016.

[16] Marinus A. Otte, et al.," Improved Biosensing Capability with Novel Suspended Nanodisks," J. Phys. Chem. C, vol. 115, pp. 5344-5351, 2011.

[17] Y. Shen, J. Zhou, T. Liu, Y. Tao, R. Jiang, M. Liu, G. Xiao, J. Zhu, Z. K. Zhou, X. Wang, C. Jin, J. Wang, "Plasmonic gold mushroom arrays with refractive index sensing figures of merit approaching the theoretical limit," Nat. Commun., vol. 4, p. 2381, Aug. 2013.

[18] George Mavrotas, "Effective implementation of the e-constraint method in Multi-Objective Mathematical Programming problems,' Applied Mathematics and Computation, vol. 213,pp. 455 - 465. 2009. 